\title{
WIKI::SCORE A collaborative environment for music transcription and publishing
}

J.J. Almeida

N.R. Carvalho

J.N. Oliveira

Ref. $[\mathrm{ACO} 11]-2011$

J.J. Almeida, N.R. Carvalho, J.N. Oliveira. Wiki::score a collaborative environment for music transcription and publishing. Information Services and Use, 31(3-4):177-187, 2011. DOI 10.3233/ISU-2012-0647. 



\title{
WIKI::SCORE
}

\section{A collaborative environment for music transcription and publishing ${ }^{1}$}

\author{
José João Almeida ${ }^{\mathrm{a}, *}$, Nuno Ramos Carvalho ${ }^{\mathrm{a}}$ and José Nuno Oliveira ${ }^{\mathrm{a}, \mathrm{b}}$ \\ ${ }^{a}$ University of Minho, Braga, Portugal \\ ${ }^{\mathrm{b}}$ HASLAb/INESC TEC \\ E-mails: \{jj, narcarvalho,jno\}@di.uminho.pt
}

\begin{abstract}
Music sources are most commonly shared in music scores scanned or printed on paper sheets. These artifacts are rich in information, but since they are images it is hard to re-use and share their content in todays' digital world. There are modern languages that can be used to transcribe music sheets, this is still a time consuming task, because of the complexity involved in the process and the typical huge size of the original documents.

WIKI: SCORE is a collaborative environment where several people work together to transcribe music sheets to a shared medium, using the notation. This eases the process of transcribing huge documents, and stores the document in a well known notation, that can be used later on to publish the whole content in several formats, such as a PDF document, images or audio files for example.
\end{abstract}

Keywords: Music transcription, ABC, wiki, collaborative work, music publishing

\section{Introduction}

Music transcriptions are most electronically shared is pictorial formats, as pictures or scans (copyright permitting) of the original music scores. Although this information is semantically rich it is hard to re-use or transform in automatic and systematic ways, without human intervention. Imagine you have a scanned book, content in images, but if you want to count the number of words in the entire book a transcription of the content would prove much more useful and easy to process. This premise is also valid for music representations: a more textual or symbolic transcription of the content, while semantically as rich as the original, is easier to re-use, transform and process. Ultimately this representation is rich enough to produce the common pictorial formats with a high level of quality and accuracy.

The problem with this type of transcription is that it is harder, more complex and time consuming than simply scanning or photographing manuscripts (books or music scores). Distributed proofreading [15] has already proven that a community driven distributed proofreading approach can ease this task. Applying a map-reduce [3] philosophy, the original manuscript is partitioned in small chunks, and the work of transcribing and validating is performed on each chunk individually. Eventually all the processed

\footnotetext{
${ }^{1}$ Paper also published in the Proceedings of the 16th International Conference on Electronic Publishing, ISBN 978-1-61499064-2.

*Corresponding author. E-mail: jj@di.uminho.pt.
} 
chunks are compiled together to build the final transcription. From this source many documents can be produced including images. Moreover, this plain text representation is easier to process and transform for performing any arbitrary task. The works of Hoos et al. [10] on musical information retrieval, Nienhuys et al. [17] on a system for automated music engraving for example, emphasize the advantages of having a more processable plain text notation. Such advantages are also defended and advocated in [22] about automatic music classification using text mining techniques.

The platform described in this article supports a large scale, collaborative transcription of music scores into a plain text symbolic representation. This representation is easy to re-use, process and transform, and can be published in a heterogenous set of different formats, MIDIs, images or PDF documents for example. Section 2 introduces relevant work and concepts. Section 3 describes WIKI::SCORE in some detail. Section 4 describes the experimental usage of the platform in a real case study. Finally, in the last sections some conclusions are driven and some ways to improve this work are described.

\section{Related work}

\subsection{Music writing applications}

Several music score authoring systems are already available, examples of the most popular being:

- Sibelius [11] is commercial desktop application provides a clear and intuitive interface to write music. It can be used to transcribe music scores, and with the use of plugins, export scores to some known formats. This application is widely used by music professionals.

- Finale [12] is another commercial desktop application that can be used to write or transcribe music, also enjoying wide popularity.

- Denemo [4] is a free software (GPL) music notation editor available for many different platforms.

- MuseScore [21] is a free cross-platform music notation program that offers a cost-effective alternative to commercial programs such as some programs described earlier.

All these applications are useful for writing music but they lack direct support for distributed, collaborative work. But nothing prevents anyone for using them to create transcriptions written in well known notations and later sharing and combining them, as some of these tools export music scores to plain text representations.

\subsection{The ABC notation}

The AвC $[7,19,25]$ notation for music transcription is based on plain text. It is very compact and with many associated tools already available. It relies essentially on a direct representation of musical elements as ASCII characters making it economic and intuitive to use. This language has a simple and clean syntax, and is powerful enough to produce professional and complete music scores. Figure 1 is a small example of a source file written in ABC. Figure 2 illustrates the image generated using the source illustrated in Fig. 1.

$A B C$ is the notation adopted for the collaborative environment described in this paper mainly for the following reasons:

- powerful enough to describe most music scores available in paper;

- actively maintained and developed; 
$\mathrm{X}: 1$

$\mathrm{M}: \mathrm{C}$

$\mathrm{L}: 1 / 4$

$\mathrm{K}: \mathrm{C}$

c2de $\mid$ A>Bc2 | f>de2 | g2de | A>Bc2 | d>ec2 | d3e | c4

Fig. 1. ABC notation example. Pitch is represented by letters and duration by numbers, relative to what is specified in the header's L field. Symbol '>' abbreviates dotted rhythm.

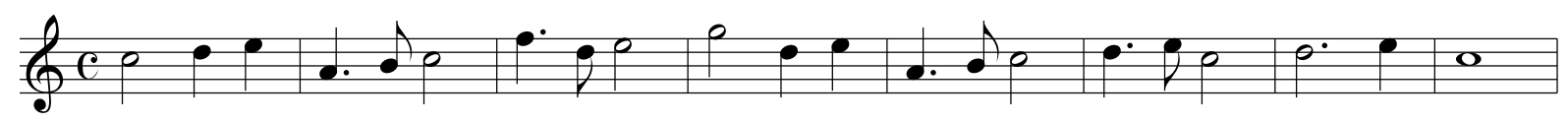

Fig. 2. Image generated from notation example in Fig. 1. First bars of the first movement of Schubert's symphony D.729.

- the source files are written in plain text files;

- there are already many tools for transforming and publishing;

- this format can be easily converted to other known formats;

- compact and clear notation;

- open source.

Other music notations are also available, LilyPond [16,17] and MusicXML [8,9] being good alternatives. The adoption of these representations were discarded in favor of $\mathrm{ABC}$ mainly because although all of them can be stored in plain text files, LilyPond and MusicXML are much more verbose and have a more cumbersome and less compact syntax, as opposed to $\mathrm{ABC}$, which is more suitable for online editing in a wiki environment. Also $\mathrm{ABC}$ is being actively extended to allow many details and tricks that are required in complex score transcription.

\subsection{Web applications: Wikis}

A Wiki [14] is basically a web site with some key features that make it more suitable for certain tasks. Central to these are tasks that provide for collaborative work, because of the multi-user content creation driven philosophy a wiki interface provides.

Some important features of wikis justifying the adoption of such a tool as a base for the environment described in this paper are:

- multi user collaborative environment - different people can add and edit content at the same time;

- content change history with version control and revert options;

- web interface, so no additional software is required other than a commonly available web browser;

- easy to extend features and syntax via plugins;

- possible to customize via template engines;

- a wiki invites users to contribute to the content;

- wiki pages are subject to the phenomenon of Darwikinism [26], which typically provides for higher quality contents [13];

- the edited content is immediately available;

- built-in lock system to prevent content corruption.

In particular, DoKUWIKI [6] was adopted because of the following reasons, implements all the features described in the previous list: 
- implements all the features described in the previous list;

- easy to install and maintain;

- the wiki content is stored in plain text files;

- it uses a clean plugin system that allows adding new features and syntax options;

- a plugin that adds ABC syntax to the wiki was already available;

- it is open source.

Wikis have already proven very useful and versatile in many scenarios, a good example being the well known Wikipedia [27], a free encyclopedia with almost 4,000,000 articles which anyone can edit or validate.

\subsection{Other projects}

A brief list of other interesting projects that in some way contributed to the development of this work follows:

- Mutopia [24] is a LilyPond based music score archive. All the provided files are free to download, print out, perform and distribute. At the time of writing, Mutopia has 1710 musics for download from Beethoven to Joplin, from Harp to Piano or full Orchestra. This project clearly helps to show how sharing transcriptions in source textual formats is an advantage.

- Noteflight [18] in an online application more in line with the work described in this paper although it lacks the collaborative driven philosophy. It allows to import and export from MusicXML.

- Distributed Proofreaders [5]. Although not related with music this project is a success case of the distributed proof reading paradigm that this work is advocating and could well be adopted in collaborative music transcription.

\section{The WIKI::SCORE Toolkit}

WIKI::SCORE is a toolkit that provides a wiki with the required features and tools to aid in collaboratively transcribing and publishing music scores. The main advantage is to make use of the typical features already provided by a wiki in music transcribing tasks.

\subsection{Workflow}

This section describes the workflow used to process a music manuscript in the WIKI::SCORE environment. Figure 3 gives an a simplified overview of the entire process.

The main idea is that the overall process starts with an original manuscript, most commonly available in paper or digitally scanned, and ends with a complete transcription of the music to ABC notation. This transcription is also used to produce a MIDI audio file, and a PDF document which contains a high quality music score. The complete $\mathrm{ABC}$ source is also provided for anyone who needs to perform any other transformation, including data mining for analysis in musicology.

\subsubsection{Project start}

The first stage when aiming to transcribe a music manuscript is to create a new project. The wiki application provides a particular page to perform this task. The process starts by providing some meta information and a name for the project, which can be updated later. The next step is to split the manuscript 


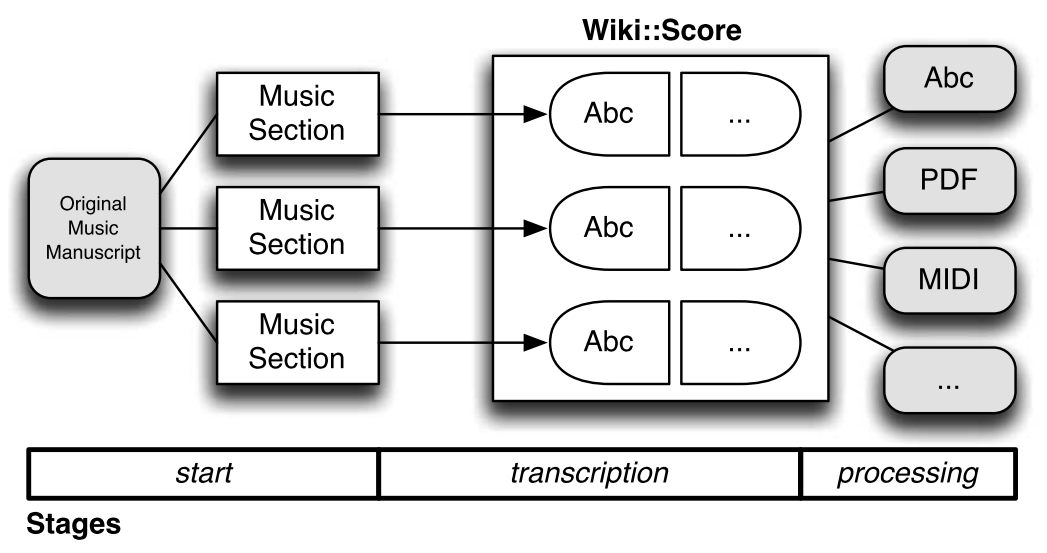

Fig. 3. WIKI::SCORE architecture overview.

in sections and parts, letting the system then create all the required wiki pages, based on default templates. Automatically using default templates, the page for every section and part is created and became immediately accessible for users to edit.

This two-dimensional array of units (sections and parts) forms a matrix that initially has no $\mathrm{ABC}$ associated with each cell. (More details about this in the sequel.)

\subsubsection{Transcription stage}

In this stage the music manuscript parts are transcribed by humans independently (one per wiki cell). This means that the corresponding $\mathrm{ABC}$ will be filled up in a cell-wise manner, starting from an initially empty matrix. Any cell (section or part) can be addressed individually and validated. Contributors are required only to access the cell they wish to transcribe and edit the corresponding wiki page, as every page is linked and easy to find.

During this stage validation tasks can be performed in parallel. These tasks can also take advantage from the wiki system, since comments and feedback can be added to individual sections' pages.

\subsubsection{Processing and publishing stage}

In this stage, that can be performed at any time, two functions are available for processing the matrix:

$$
\begin{aligned}
& \text { process : Matrix } \times \text { Cut } \times \text { Template } \longrightarrow A b c \\
& \text { publish }: \text { Abc } \times \text { Format } \longrightarrow \text { File }
\end{aligned}
$$

The process function is responsible for compiling an $\mathrm{ABC}$ document for a given project. To build this, the function operates over the project Matrix, and a Cut matrix can be used to process a smaller subset of the entire project. This function also uses a Template that can change the final ABC generated. These files written in $\mathrm{ABC}$ can then be used by the publish function to produce a document in a given Format, for example an PDF or a MIDI file.

It is possible to publish the entire document or a small part of it, as this proves useful for proof-reading tasks for example. For this purpose, simple matrix operations are used. A row in this matrix is associated with a part (instrument, voice) and a column corresponds to a piece (e.g. a movement of a symphony, the overture of an opera etc.) The matrix algebra is such that multiplying by 1 means selection and by 0 means discarding. As explained in Fig. 4 the Cut matrix can be regarded as a pair of vectors $v 1$ (resp. $v 2$ ) selecting parts (resp. pieces). The matrix additive operation corresponds to concatenation. Equation (1) 


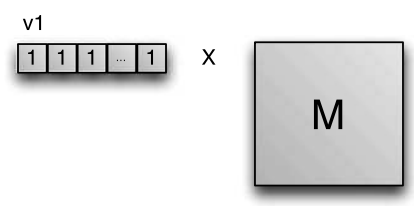

(a)

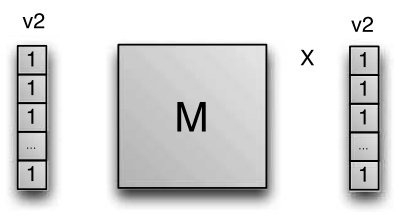

(b)

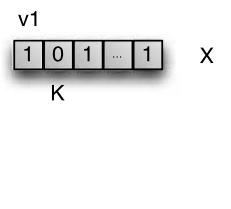

(c)

Fig. 4. Matrix multiplying examples. Row vector $v 1$ works part-wise; column vector $v 2$ works piece-wise. (a) Conductor's score. (b) All individual parts. (c) All parts but $k$.

shows how to publish a single part just by multiplying the matrix with a vector which selects the first part (the only 1 in the vector).

$$
\begin{gathered}
\left(\begin{array}{llll}
1 & 0 & \cdots & 0
\end{array}\right) \times\left(\begin{array}{ccccc}
A b c_{1,1} & A b c_{1,2} & \cdots & A b c_{1, n} \\
A b c_{2,1} & A b c_{2,2} & \cdots & A b c_{2, n} \\
\vdots & \vdots & \ddots & \vdots \\
A b c_{p, 1} & A b c_{p, 2} & \cdots & A b c_{p, n}
\end{array}\right) \\
=\left(\begin{array}{llll}
A b c_{1,1} & A b c_{1,2} & \cdots & A b c_{1, n}
\end{array}\right) .
\end{gathered}
$$

Equation (2) shows the effect of selecting all parts. Each element in the resulting vector joins all the parts for each section, See Eq. (3).

$$
\begin{gathered}
\left(\begin{array}{llll}
1 & 1 & \cdots & 1
\end{array}\right) \times\left(\begin{array}{cccc}
A b c_{1,1} & A b c_{1,2} & \cdots & A b c_{1, n} \\
A b c_{2,1} & A b c_{2,2} & \cdots & A b c_{2, n} \\
\vdots & \vdots & \ddots & \vdots \\
A b c_{p, 1} & A b c_{m, 2} & \cdots & A b c_{p, n}
\end{array}\right) \\
=\left(\begin{array}{llll}
\text { Section }_{1} & \text { Section }_{2} & \cdots & \text { Section }_{n}
\end{array}\right) \\
\text { Section }_{i}=A b c_{1, i}+\text { Abc }_{2, i}+\cdots+\text { Abc }_{p, i}
\end{gathered}
$$

The conductor's score, Section $_{1}+\cdots+$ Section $_{n}$, is obtained by multiplying $\left(\right.$ Section $_{1} \cdots$ Section $\left._{n}\right) \times$ $(1 \cdots 1)$.

Using this approach, it is simple to process or publish the intended elements of the matrix. Figure 4 illustrates some other examples that can be used to produce typical scores.

\subsection{Web interface}

A wiki is a web application. So any common browser or equivalent tool is enough for collaborating in WIKI::SCORE transcription tasks. Typically there is a wiki page for each project, which contains some meta information (original manuscript name, author, etc.) and links to other relevant pages. One important section in this index page is the matrix of links available to access each music section and voice. This matrix is illustrated in Fig. 5.

From this matrix any contributor may access each cell in the matrix, corresponding to a particular part (e.g. instrument) and section (e.g. aria of an opera). There is a specific page for each cell where the ABC transcription can be made, and since this is a wiki much more information can be added, notes about validation and error issues for example. The edition page is illustrated in Fig. 6. 


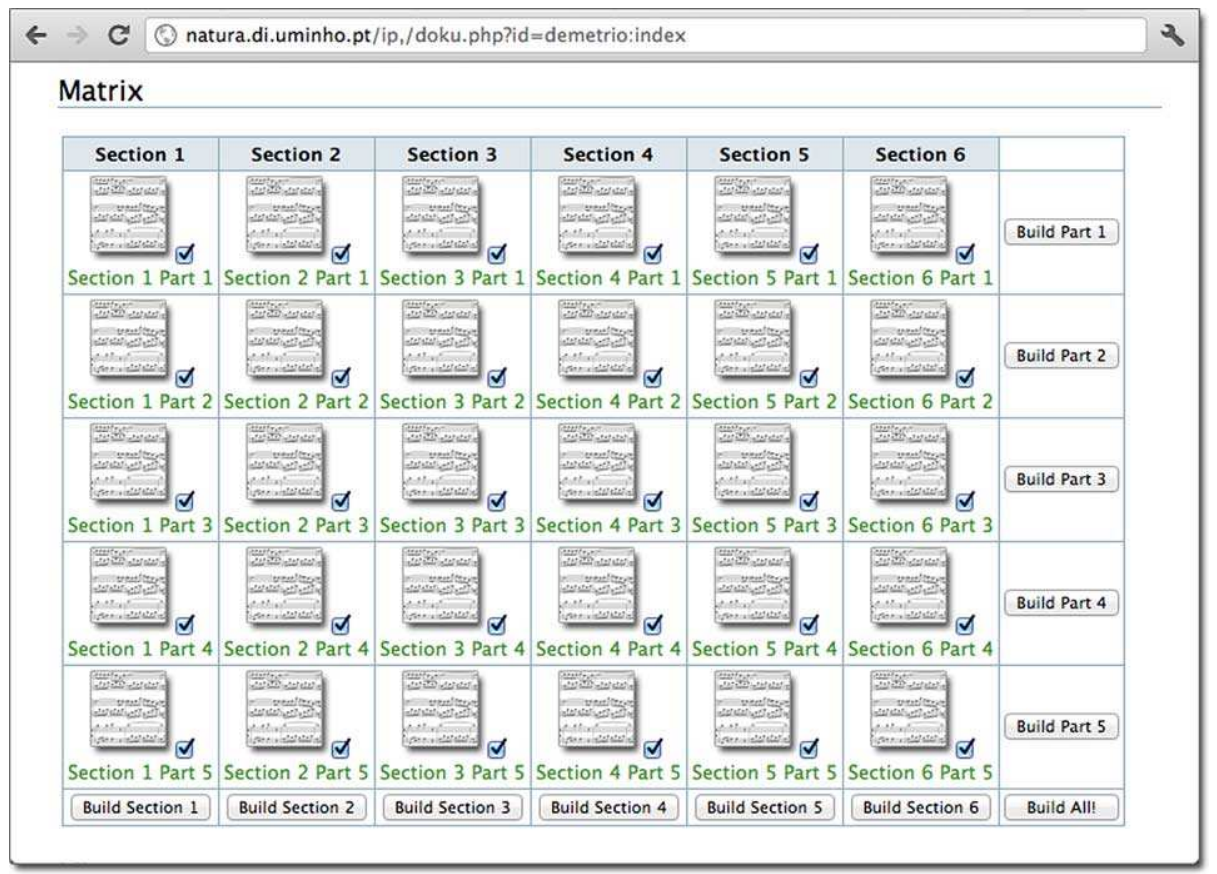

Fig. 5. Matrix of sections and parts that make up the entire music. The "Build" buttons implement the matrix selection mechanism. (Colors are visible in the online version of the article; http://dx.doi.org/10.3233/ISU-2012-0647.)

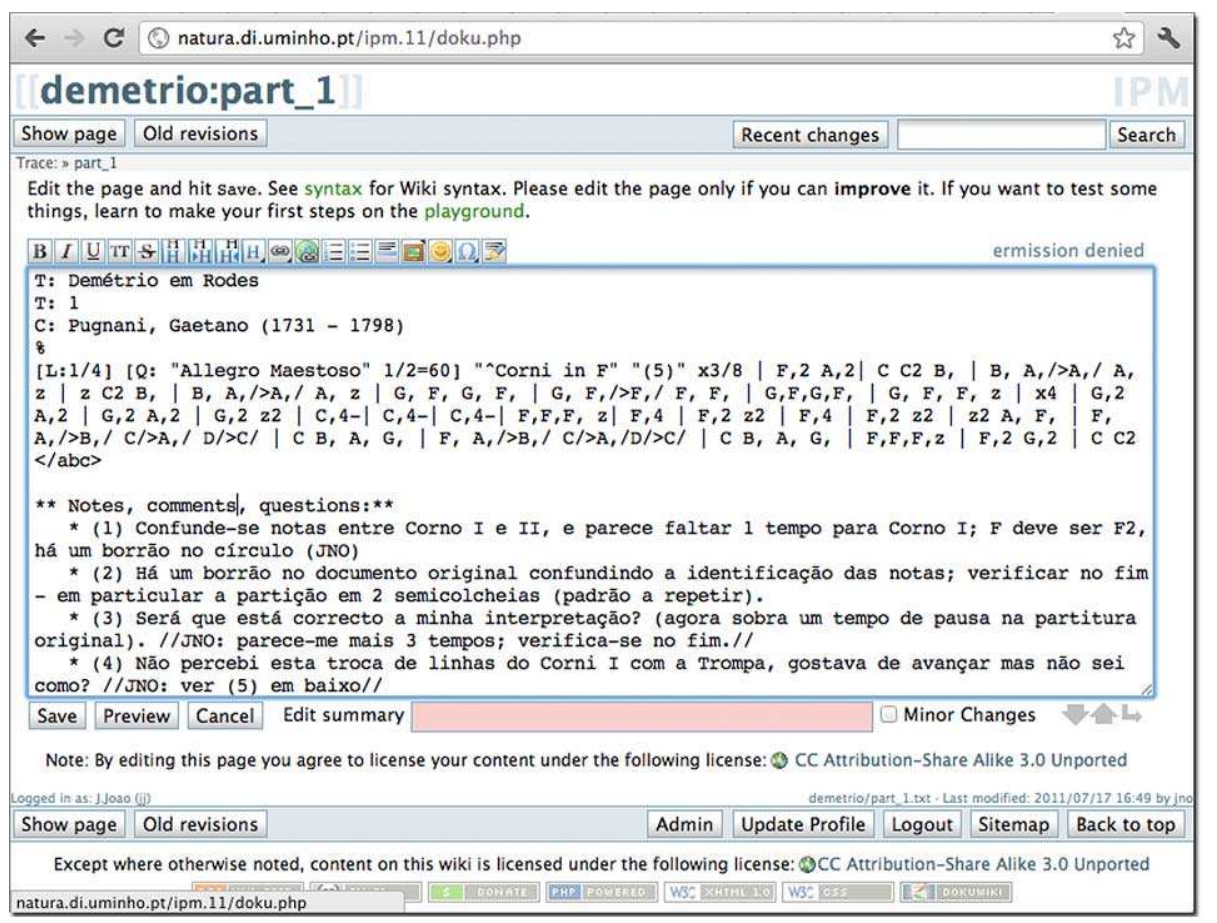

Fig. 6. Editing. (Colors are visible in the online version of the article; http://dx.doi.org/10.3233/ISU-2012-0647.) 
The project index page provides links for documents published in several formats, built from the ABC notation:

- complete transcription in $\mathrm{ABC}$ format, compiled from all the transcriptions in the matrix;

- PDF document containing a high quality pictorial representation of the music score;

- a MIDI file that can be used to hear the transcribed music.

These items are also provided for each cell in the matrix, in the corresponding wiki page. This is useful while editing and when performing validation tasks.

\section{Experimental validation}

The WIKI::SCORE prototype ${ }^{1}$ has been used to support the transcription in modern notation of an Italian 18c opera manuscript: Demetrio a Rodi: Dramma per Musica: Rappresentado nel Regio Teatro di Torino nella Primavera del 1789, by Gaetano Pugnani (1731-1798). The libretto by Giandomenico Boggio and Giuseppe Banti was published by Presso O. Derossi, 1789, a digitalized version of which is available from the public Bayerischen Staatsbibliothek [1].

The original music manuscript was found in Fundo do Conde de Redondo and is available online from the Biblioteca Nacional Digital [20]. To the best of the authors' knowledge, there is no book edition of the score.

Some properties of this project are:

- the manuscript has 18 parts (4 voice choir +14 instrumental parts);

- the main divisions - Overtura (40 pages), Atto primo (149 pages), Atto secondo (221 pages) - are very big;

- the manuscript presents some paleographic challenges;

- $18 \mathrm{c}$ music notation conventions have to be understood and reproduced in modern notation;

- the extant manuscript is the conductor's score, full of abbreviations, calling for the necessary adaptation in order to produce a document readable by a common musician;

- the final result should be a modern-notation standard edition.

An instance of this environment was made available to the students of a BSc degree in music. ${ }^{2}$ The subject of their work in the lab sessions and home work was "the first column of the matrix": the Overtura of the opera. All students were used to systems such as Sibelius [11] and no one had worked on a wiki platform before. Their reaction in the beginning was negative, as they thought they would do the job faster using the systems they had used before. Gradually, they became aware of the advantages of cooperative work, in particular when they realized how easy it was for their teachers to correct their mistakes overnight, as if by magic. One student at some stage lost most of her work in a ill-fated edition session. She was about to start all over again when the teacher recalled that there was a version control behind the scene and nothing had been lost. This remains one of the most interesting episodes of the course, showing how laptops force people to work in isolation, ignoring all that has been achieved meanwhile in cooperative contents editing.

Figure 7 illustrates the first page of the published final version in PDF format. This corresponds to the manuscript score depicted in Fig. 8.

\footnotetext{
${ }^{1}$ http://wiki-score.org.

${ }^{2}$ Computing for Musicology course, 2nd year of the University of Minho's degree in Music, academic year 2010/2011.
} 


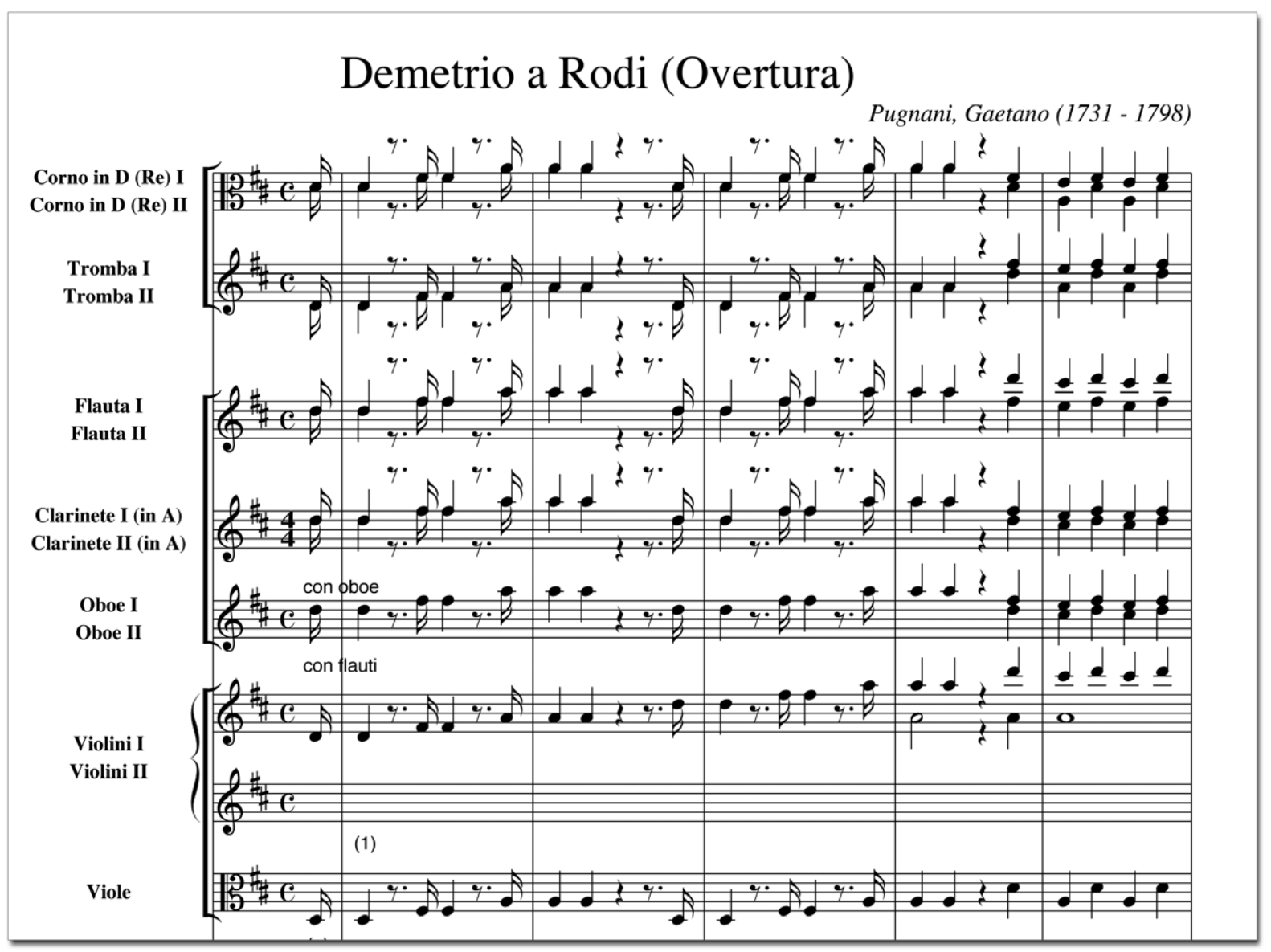

Fig. 7. Published PDF document excerpt.

\section{Conclusion}

$\mathrm{ABC}$ is a simple yet powerful notation for transcribing music sheets which interacts with several tools that later allow to publish contents in different well known formats, as PDF or MIDI files.

As $\mathrm{ABC}$ source files are plain text, it is easy to take advantage of a common collaborative work environment - a wiki. This allows for people to get into the system and interact with each other, contributing with ad-hoc work without having to learn to use other tools or buy expensive WYSIWYG tools.

Putting everything together based on a small set of glueing functions proves an excellent way to transcribe huge manuscript scores in small time spans, in a collaborative way. Also aiming at a more symbolic representation has proven useful since the same typical documents could be produced (images or MIDI), but this representation is easier to transform and process in more automated and systematic ways.

Experimental cooperative work in this area introduces a new challenge related with the bi-dimensional structure of multi-part music: synchronization. Thus the horizontal dimension of the matrix had to be introduced, the notion of reference part being crucial for proof-reading. The ABC matrix often suffers from stability issues, mainly due to synchronization problems. A simple error in the duration of a note 


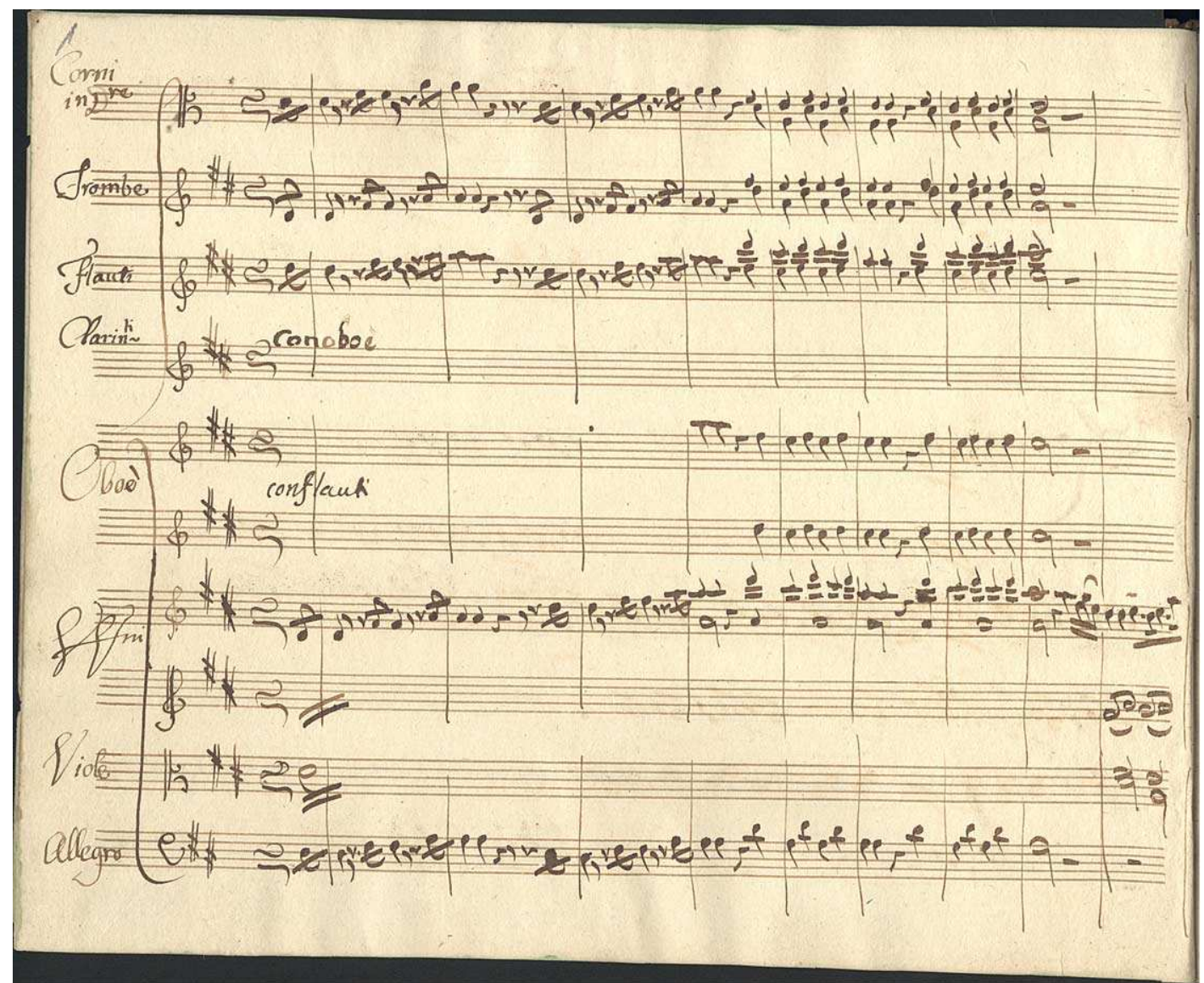

Fig. 8. Folio 1 of the manuscript score of the Overtura of the opera Demetrio a Rodi. (Available from [2].) (Colors are visible in the online version of the article; http://dx.doi.org/10.3233/ISU-2012-0647.)

in a part is enough to create huge synchronization problems. This is why it is important to be able to de-select (problematic) parts until they are corrected.

\subsection{Future work}

There are several features that could improve this work, among others:

- allow the edition of default templates for several operations, including generating the final $\mathrm{ABC}$ transcript;

- visual editor for cell editing for non-proficient $\mathrm{ABC}$ contributors;

- cooperative building of style books, gathering knowledge from music paleontologists;

- produce all-but-one MIDI files which musicians can use at home when training their part (already technically possible);

- development of a Domain Specific Language to allow a more accurate control over the project structure and synchronization.

We believe the interference implicit in the bi-dimensional structure of music contents presents new challenges in collaborative editing. Open Source communities have a lot of experience in this aspect (as 
computer programs are also made of interfering parts) and learning with them could be interesting in the future.

\section{Acknowledgements}

The authors would like to thank Biblioteca Nacional Digital for the excellence of their work in making music manuscripts available to the general public. They would also like to thank the reviewers for their valuable insight and detailed comments, which aided in improving this paper.

\section{References}

[1] Bayerischen Staatsbibliothek, available at: http://www.bsb-muenchen.de/ (last accessed: 26-03-2012).

[2] Biblioteca Digital Nacional. Demetrio a Rodi, available at: http://purl.pt/16734/1/P1.html (last accessed: 26-03-2012).

[3] J. Dean and S. Ghemawat, Mapreduce: Simplified data processing on large clusters, Communications of the ACM 51(1) (2008), 107-113.

[4] Denemo, available at: http://www.denemo.org/ (last accessed: 18-01-2012).

[5] Distributed proofreaders, available at: http://www.pgdp.net/ (last accessed: 18-01-2012).

[6] A. Gohr, Dokuwiki, available at: http://www.dokuwiki.org/ (last accessed: 18-01-2012).

[7] G. Gonzato, Making Music With Abc Plus, 2011, available at: http://abcplus.sourceforge.net/\#ABCGuide (last accessed: 18-01-2012).

[8] M. Good, Musicxml for notation and analysis. The Virtual Score: Representation, Retrieval, Restoration 12 (2001), 113124.

[9] M. Good, MusicXML: An internet-friendly format for sheet music, in: XML Conference \& Exposition, Orlando, FL, 2001.

[10] H. Hoos, K. Renz and M. Görg, Guido/mir-an experimental musical information retrieval system based on guido music notation, in: International Symposium on Music Information Retrieval, Bloomington, IN, USA, 2001.

[11] Inc. Avid Technology. Sibelius, available at: http://www.sibelius.com/ (last accessed: 18-01-2012).

[12] Inc. MakeMusic, Finale, available at: http://www.finalemusic.com/ (last accessed: 18-01-2012).

[13] B. Lamb, Wide open spaces: Wikis, ready or not, EDUCAUSE Review 39 (2004), 36-49.

[14] B. Leuf and W. Cunningham, The Wiki Way: Quick Collaboration on the Web, Addison Wesley, 2001.

[15] G.B. Newby and C. Franks, Distributed proofreading, in: Proceedings of the Joint Conference on Digital Libraries, IEEE, 2003, pp. 361-363.

[16] H.-W. Nienhuys, LilyPond, automated music formatting and the art of shipping, in: Forum Internacional Software Livre (FISL7.0), Porto Alegre, Brazil, 2006.

[17] H.W. Nienhuys and J. Nieuwenhuizen, Lilypond, a system for automated music engraving, in: Proceedings of the XIV Colloquium on Musical Informatics (XIV CIM 2003), Citeseer, 2003, pp. 167-172.

[18] Noteflight LLC. Noteflight, available at: http://www.noteflight.com/ (last accessed: 18-01-2012).

[19] I. Oppenheim, The ABC Music standard 2.0 (December 2010), 2010, available at: http://abcnotation.com/wiki/ abc:standard:v2.0 (last accessed: 18-01-2012).

[20] G. Pugnani, Demetrio a rodi - Dramma per musica: Rappresentado nel regio teatro di Torino nella primavera del 1789. Digital manuscript music, Biblioteca Nacional Digital, Fundo do Conde de Redondo, available at: http://purl.pt/ 16734/1/P5.html (last accessed: 18-01-2012).

[21] W. Schweer, Musescore, available at: http://www.musescore.org/ (last accessed: 18-01-2012).

[22] A. Simões, A. Lourenço and J.J. Almeida, Mining classical music score for epoch classification, in: New Trends in Artificial Intelligence, J. Neves, M.F. Santos and J.M. Machado, eds, Guimarães, Portugal, December 2007, pp. 791-799.

[23] A.M. Simões and J.J. Almeida, Music publishing, in: ElPub 2003 - International Conference on Electronic Publishing, S. Costa et al., eds, Universidade do Minho, Guimarães, June 2003, pp. 288-298.

[24] The mutopia project, available at: http://www.mutopiaproject.org/ (last accessed: 18-01-2012).

[25] C. Walshaw, Abc notation, available at: http://abcnotation.com/ (last accessed: 18-01-2012).

[26] Wikimedia, Darwikinism, available at: http://meta.wikimedia.org/wiki/Darwikinism (last accessed: 16-03-2012).

[27] Wikimedia Foundation. Wikipedia, available at: http://www.wikipedia.org/ (last accessed: 18-01-2012). 